\title{
On the Truth-Conditional Relevance of Modes of Presentation
}

\author{
Massimiliano Vignolo \\ University of Genoa \\ DOI: $10.2478 /$ disp-2013-0005 \\ BIBLID [0873-626X (2013) 35; pp. 57-66]
}

\begin{abstract}
Anne Bezuidenhout 1996 presents an argument for the claim that modes of presentation associated with referential terms are truth-conditionally relevant. I argue that her argument is flawed in light of the very same view on the interplay between reference and pragmatics she endorses.
\end{abstract}

Keywords

Modes of Presentation, Reference, Pragmatics, Truth Conditions.

\section{Introduction}

Referential terms contribute their referents to the truth conditional content of the propositions expressed. Many theorists hold that referential terms are associated with modes of presentation, which are ways of thinking of their referents. Modes of presentation serve to explain (i) the meaningfulness of sentences containing referential terms lacking a referent, (ii) the difference in informativeness of sentences containing co-referring terms and (iii) the truth-conditions of propositional attitude reports. For example an utterance of 'he is F' is meaningful even if the pronoun 'he' has no referent in the context of utterance, say because the speaker is having a hallucination. In that context no proposition is expressed but the utterance is not meaningless since competent speakers know the linguistic mode of presentation - the character - associated with the pronoun 'he'. Consider another example: 'this boy' and 'that boy' might have the same referent, say in a context in which 'this boy' refers to a boy currently visually presented to the speaker and the hearer and 'that boy' refers to the same boy under a different guise the speaker and

Disputatio, Vol. V, No. 35, May 2013

Received: 28/05/2012. Revised: 03/08/2012. Accepted: 06/08/2012 
the hearer met the day before. In such context the following two sentences:

(a) This boy is this boy.

(b) This boy is that boy.

might differ in informativeness, because one might not realize that the boy currently visually presented to him is the same boy as the boy one met the day before. Similarly, it might be true that John believes that this boy is this boy and false that John believes that this boy is that boy.

Modes of presentation might be linguistic or psychological. In order to explain the meaningfulness of the sentence 'he is F' in the context in which the speaker is hallucinating a referent it is sufficient to invoke the linguistic mode of presentation associated with 'he', i.e. the salient male. On the other hand, in order to explain the difference in informativeness between utterances of (a) and (b) it is necessary to invoke psychological modes of presentation, i.e. the current visual appearance of the boy and the memory of the boy the speaker and the hearer met the day before in different circumstances. According to Anne Bezuidenhout's view (1996) on the interplay between reference and pragmatics, psychological modes of presentation are associated with referential terms like 'this boy' and 'that boy' through pragmatic processes that exploit contextually available information.

Most theorists hold that modes of presentation are truth conditionally irrelevant. Their conceptual content does not affect the truth-conditions of the propositions expressed. For example, if John says 'I am F' the fact that John is the speaker of the token 'I' is not part of the truth-conditions of what John says. What John says is true in all possible worlds in which John is F independently of whether John is speaking or not. Likewise (a) and (b) have the same truthconditional content although they differ in assertability conditions and informativeness.

There are at least two ways of implementing the truth-conditional irrelevance of modes of presentation into a semantic theory. Some theorists identify the proposition expressed with the truthconditional content and then distinguish the proposition expressed from the complete content of an utterance, which is taken to include 
modes of presentation. Other theorists distinguish the proposition expressed, which includes modes of presentation, from the truthconditional content. Nothing substantive derives from the choice of one version over the other.

\section{Bezuidenhout's argument}

Anne Bezuidenhout 1996 accepts the view that modes of presentation are components of the propositions expressed and argues that modes of presentation are truth conditionally relevant. She gives the following argument. Consider the pair of sentences (c) and (d):

(c) If this boy is this boy, then John will show surprise.

(d) If this boy is that boy, then John will show surprise.

Bezuidenhout holds that we are strongly inclined to take (c) as false and (d) as true. Presumably John will not show surprise as a consequence of an instance of the principle of the reflexivity of identity. In order to do justice to our inclination, Bezuidenhout says, one needs to ascribe different truth-conditions to (c) and (d). Given that there is no difference between (c) and (d) at the level of reference and the only difference resides at the level of the modes of presentation that are associated with the expressions 'this boy' and 'that boy', the conclusion follows that modes of presentation are truth conditionally relevant.

Bezuidenhout concedes that the theorists who deny the truthconditional relevance of modes of presentation might have a rejoinder. The truth-conditional irrelevance of modes of presentation does not imply that modes of presentation cannot have an effect on the truth-conditions of certain compound sentences, in particular of sentences containing that-clauses. If one chooses the view that the propositions expressed include modes of presentation, one can hold that that-clauses refer to propositions containing modes of presentation (alternatively one can hold that that-clauses refer to complete contents - quasi-singular propositions - formed of propositions and modes of presentation). Therefore modes of presentations are among the constituents of the referents of that-clauses and as such they have a truth-conditional effect on sentences of propositional attitudes re- 
ports.

Following this line of reasoning, one can argue that (c) and (d) have the form 'If this boy is this (that) boy, then John will show surprise at that', which makes reference to the proposition - or quasisingular proposition - expressed in the antecedent. Given that the antecedents of (c) and (d) express different propositions - or quasisingular propositions - since the mode of presentation associated with the expression 'this boy' is different from the mode of presentation associated with the expression 'that boy', the truth-conditions of (c) and (d) are different and the difference in truth-value is explained without appealing to the truth-conditional relevance of modes of presentation, apart from their effect on the referents of that-clauses. Suppose the boy is Jimmy, (c) and (d) have the following truth-conditions:

'If this boy is this boy, then John will show surprise at that' is true iff Jimmy $=$ Jimmy $\rightarrow$ Be_surprised $($ John, $\langle m, m$, Jimmy, $=$ Jimmy $\rangle)$.

'If this boy is that boy, then John will show surprise at that' is true iff Jimmy $=$ Jimmy $\rightarrow$ Be_surprised(John, $\left\langle m, m^{*}\right.$, Jimmy, $=$, Jimmy $\rangle$ ).

where $m$ and $m *$ are the modes of presentation associated with the expressions 'this boy' and 'that boy'. However Bezuidenhout claims she can provide another example showing two sentences with intuitively different truth values which cannot be accounted for in the same way as the previous case. Consider the following two sentences:

(e) If the boy can lift this, John will think the boy is strong.

(f) If the boy can lift that, John will think the boy is strong.

Bezuidenhout envisages the following scenario as the context of utterance. The demonstratives 'this' and 'that' refer to the same dumbbell-shaped piece of Styrofoam. Viewed from one visual perspective the fake dumbbell looks like a genuine dumbbell, and viewed from another visual perspective it looks like a piece of Styrofoam. The speaker, the hearer and John are all present in this conversational context and have similar visual perspectives on the boy and the dumbbell-shaped piece of Styrofoam. So the propositional attitude 
reports are interpreted in the opaque rather than in the transparent sense. Bezuidenhout claims that in this scenario we are strongly inclined to take (e) as true - when the visual appearance of the fake dumbbell is that of a genuine dumbbell - and (f) as false - when the visual appearance of the fake dumbbell is that of a piece of Styrofoam.

Bezuidenhout considers and rejects a rejoinder of the same kind as the previous one. One may be tempted to say that (e) and (f) have the form 'If the boy can lift this (that) and John is aware of that, then John will think the boy is strong' which makes reference to the proposition the boy can lift this in (e) and to the proposition the boy can lift that in (f), which are different propositions because, as said above, the modes of presentation associated with the demonstratives 'this' and 'that' are different.

Bezuidenhout (1996: 153) refutes this rejoinder because she says that in order to understand (e) and (f) as material conditionals there is no need to understand John's awareness of the event in the antecedent to have been asserted in (e) and (f). The truth conditional content of (e) and (f) does not make reference to John's awareness that the boy can lift this(that) and the solution that is workable for the difference in truth value between (c) and (d) is not suitable for explaining the difference in truth value between (e) and (f). Thus, Bezuidenhout concludes - and I agree with her on this point - that (e) and (f) do not have the following truth-conditions, where $m$ is the mode of presentation associated with 'the boy' and $m$ ' and $m *$ are the modes of presentation associated with 'this' and 'that' and $\mathrm{O}$ is the dumbbell-shaped piece of Styrofoam:

'If the boy can lift this, then John will think the boy is strong' is true iff Can_lift(Jimmy, O) $\wedge$ Aware(John, $\left\langle m, m^{\prime}\right.$, Jimmy, Can_ lift, $O\rangle) \rightarrow$ Think(John, $\langle m$, Jimmy, Being_strong $\rangle)$

'If the boy can lift that, then John will think the boy is strong' is true iff Can_lift(Jimmy, O) $\wedge$ Aware(John, $\left\langle m, m^{*}\right.$, Jimmy, Can_ lift, $O\rangle) \rightarrow$ Think(John, $\langle m$, Jimmy, Being_strong $\rangle)$. 


\section{Counterargument}

I hold that Bezuidenhout's argument is flawed. My counterargument to Bezuidenhout is that if one accepts Bezuidenhout's view on the interplay between reference and pragmatics, then one can hold that the that-clause 'that the boy is strong' refers to two different propositions when embedded in (e) and when embedded in (f). The propositions referred to are different because they contain different modes of presentation of Jimmy. Therefore, (e) and (f) differ in truth conditional content, and this explains their divergence in truth value. My claim, then, is that if Bezuidenhout's view on the interplay between reference and pragmatics is correct, then one is not forced to accept the truth-conditional relevance of modes of presentation in Bezuidehnout's strong sense which goes beyond their effect on the truth-conditions of propositional attitudes reports in order to account for the difference in truth value between (e) and (f).

One of the main points in Bezuidenhout's view is that reference is mediated by psychological modes of presentation. Psychological modes of presentation depend on contextually available information whose elaboration goes through pragmatic processes. For example the demonstrative 'this' in (e) and the demonstrative 'that' in (f) refer to the same piece of Styrofoam. But the modes of presentation with which they are associated are different. One presents the piece of Styrofoam as a genuine dumbbell, the other as a fake dumbbell.

Sentence (e) and sentence (f) make reference to the same piece of Styrofoam under different modes of presentation, and they make reference to the same boy, i.e. Jimmy, as well. I think one may grant that the first occurrence of the expression 'the boy' in (e) and the first occurrence of the same expression in (f) are associated with the same mode of presentation. But there is no reason why one ought to accept that the first occurrence and the second occurrence of 'the boy' in (e) are associated with one and the same mode of presentation, and that the second occurrence of 'the boy' in (e) and the second occurrence of 'the boy' in (f) are associated with one and the same mode of presentation. The antecedents in (e) and (f) create two different linguistic contexts, and one can hold that the modes of presentation of the second occurrences of 'the boy' in (e) and (f) depend on such linguistic contexts, as linguistic contexts are part of 
the contextually available information. One might suppose that the mode of presentation $(m)$ associated with the second occurrence of 'the boy' in (e) is something like boy who can lift this dumbbell, whereas the mode of presentation $\left(m^{*}\right)$ associated with the second occurrence of 'the boy' in (f) is something like boy who can lift that piece of Styrofoam. The truth-conditions of (e) and (f) are the following:

'If the boy can lift this, John will think the boy is strong' is true iff Can_lift(Jimmy, O) $\rightarrow$ Think(John, $\langle m$, Jimmy, Being_strong $\rangle$ ).

'If the boy can lift this, John will think the boy is strong' is true iff Can_lift(Jimmy, O) $\rightarrow$ Think(John, $\left\langle m^{*}\right.$, Jimmy, Being_strong \rangle .

(e) says that if Jimmy can lift $O$, then John will think of Jimmy that he is strong under the mode of presentation $m$, i.e. boy who can lift this dumbbell and (f) says that if Jimmy can lift O, then John will think of Jimmy that he is strong under the mode of presentation $m^{*}$, i.e. boy who can lift that piece of Styrofoam. This analysis accords with the intuition that (e) is true and (f) is false. If this analysis is correct, (e) and (f) turn out to have different truth conditional contents because the that-clauses in their consequents make reference to propositions that are different as containing different modes of presentation. Bezuidenhout's right intuition that (e) and (f) diverge in truth value can be accommodated within the view that modes of presentation have a truth conditional effect in propositional attitudes reports without being truth conditionally relevant in the strong sense Bezuidenhout claims. ${ }^{1}$

${ }^{1}$ An anonymous referee of this journal commented that a better way of responding was by pointing out that 'thinking', in the relevant context, is 'thinking on some basis', and hence (e) and (f), properly expanded, would go like this:

(e) If the boy can lift this, John will think on that basis that the boy is strong.

(f) If the boy can lift that, John will think on that basis that the boy is strong.

The 'that basis' would refer to the proposition expressed by the antecedent, and hence this would be a reply along the lines of the one discussed for the argument based on (c) and (d). I agree with the anonymous referee that this explains the difference in truth value of (e) and (f). However, his solution does not accommodate Bezuidenhout's constraint that the causal relation between the event 
I close this note with my answer to an objection that has been raised to my counterargument. I argued that the two occurrences of 'the boy' embedded under attitude ascriptions express different modes of presentation that are parasitic on the modes of presentation expressed by 'this' and 'that'. I do not claim that modes of presentation associated with singular terms always change when the singular terms are embedded under the scope of logical operations, like conditional constructions, and attitude ascriptions. My view is that modes of presentation might change in those circumstances. The linguistic contexts in which singular terms are embedded form a source of contextual information that might be relevant for building up the modes of presentation of the embedded singular terms according to the very same view that Bezuidenhout endorses on the interplay between singular reference and pragmatics. However, the objection goes, to hold that modes of presentation might change is sufficient for raising the following problem. Suppose, for instance, that it has been established that John will ring the bell if he thinks that the boy is strong. We can reason about whether John will ring the bell under various situations: if the boy can lift this, then John will think that the boy is strong. Therefore, if the boy can lift this, John will ring the bell. If the occurrences of 'the boy' are associated with different modes of presentation, it is unclear why we can infer correctly that conclusion. In other words, it is unclear how we can account for the validity of the following inference:

1. If John thinks the boy is strong, John will ring the bell.

2. If the boy can lift this, John will think the boy is strong.

Therefore

3. If the boy can lift this, John will ring the bell.

We can formalise the inference. Let Q be the bell, $m$ ' the mode of presentation associated with the occurrence of 'the boy' in 1. and in the antecedent of 2., m" the mode of presentation associated with the occurrence of 'the boy' in the that-clause in 2., $p$ the proposition $\langle m$ ', Jimmy, Being_strong $\rangle$ and $p^{*}$ the proposition 〈m", Jimmy, Being_strong $\rangle$ : 
1. Think(John, $p) \rightarrow \operatorname{Ring}(J o h n, Q)$.

2. Can_lift(Jimmy, O) $\rightarrow$ Think(John, $p *)$.

Therefore

3. Can_lift(Jimmy, O) $\rightarrow \operatorname{Ring}(J o h n, Q)$.

The inference is clearly not valid and the reason is that $p \neq p *$ because $m^{\prime} \neq m$ ', which is exactly what my view predicts.

I reply to the above objection that the incompatibility of my view and the intuitive validity of the inference 1 . to 3 . is only apparent. The inference 1. to 3 . is enthymematic, it has a suppressed premise. The suppressed premise is the following, which I take to be true:

Think(John, $\left.p^{*}\right) \rightarrow$ Think(John, $\left.p\right)$.

By assumption, $m$ ' is a de re mode of presentation under which Jimmy is presented to the speaker (and to John as well in Bezuidenhout's scenario). This is to say that m' makes Jimmy salient for reference. It is true that $m$ " is different from $m$ ', but $m$ " is a completion of $m$ '. Suppose $m$ ' is boy wearing a red $t$-shirt. Then, $m$ "' will be boy wearing a red $t$-shirt who can lift this dumbbell. If $m$ ' is enough for making Jimmy salient for reference, anyone who thinks of Jimmy under the mode of presentation boy wearing a red $t$-shirt who can lift this dumbbell will be able to think of Jimmy under the mode of presentation boy wearing a red $t$-shirt. Therefore, anyone who thinks of Jimmy that he is strong under the mode of presentation boy wearing a red $t$-shirt who can lift this dumbbell will be able to think of Jimmy that he is strong under the mode of presentation boy wearing a red t-shirt. In general, if an agent thinks of an object under a mode of presentation $m *$ that is a completion of another mode of presentation $m$, which is sufficient for making the object salient for reference, then the agent is able to think of the same object under the mode of presentation $m$. For example, if agent A thinks of London as the capital of England having more than ten million of inhabitants, he will be able to think of London as the capital of England. And if A believes of London that it is North of Paris under the mode of presentation the capital of England having more that ten million of inhabitants, we can ascribe to him the belief that London is North of Paris under the mode of presentation the capital of England.

The above inference turns out to be valid if we make the suppressed premise explicit. 
1. Think(John, $p) \rightarrow \operatorname{Ring}(J o h n, Q)$.

2. Can_lift(Jimmy, O) $\rightarrow \operatorname{Think}\left(J o h n, p^{*}\right)$.

3. Think $\left(J o h n, p^{*}\right) \rightarrow \operatorname{Think}(\mathrm{John}, p)$.

Therefore

4. Can_lift(Jimmy, O) $\rightarrow \operatorname{Ring}(J o h n, Q)$.

There is no incompatibility between the view underlying my counterargument to Bezuidenhout and the validity of certain intuitive inferences.

Massimiliano Vignolo

\section{DAFIST University of Genoa} Via Balbi 4, 16124 IT Italy maxi@nous.unige.it

\section{References}

Bezuidenhout, Anne. 1996. Pragmatics and Singular Reference. Mind and Language 11:133-159. 